\title{
Optimal modification of poly(vinylidene fluoride) membrane surface by using surface-modifying macromolecules for application in membrane distillation
}

\author{
Reshad Shoaie ${ }^{\mathrm{a}}$, Javad Karimi-Sabet ${ }^{\mathrm{b}, *}$, Seyed Mohammad Ali Mousavian ${ }^{\mathrm{a}}$, \\ Parissa Khadiv-Parsi ${ }^{a}$, Rasoul Moradi ${ }^{a}$ \\ ${ }^{a}$ College of Chemical Engineering, School of Engineering, University of Tehran, Tehran, Iran, Tel. +982177797714; \\ email:r.shoae@gmail.com (R. Shoaie) \\ Tel.+982166498982; email: moosavian@ut.ac.ir (S.M.A. Mousavian), Tel.+982166498982; email: kparsi@ut.ac.ir (P. Khadiv-Parsi), \\ Tel.+982166957786; email: rmoradi@ut.ac.ir (R. Moradi) \\ ${ }^{b}$ NFCRS, Nuclear Science and Technology Research Institute, Tehran, Iran, Tel. +982188221117; email: j_karimi@alum.sharif.edu
}

Received 3 June 2016; Accepted 23 October 2016

\section{A B S T R A C T}

The optimal engineering of the surface properties was performed for poly(vinylidene fluoride) (PVDF) membrane with hydrophobic surface-modifying macromolecules (SMMs). For this aim, new hydrophobic SMM additives were synthesized using polyurethane chemistry and characterized for water contact angle, fluorine content and average molecular weight. Both modified PVDF/SMM and neat PVDF membranes were prepared through phase inversion method in a single casting step and characterized by means of scanning electron microscope, energy-dispersive X-ray spectroscopy and contact angle goniometry. The optimum preparation conditions were determined using response surface methodology. The effects of the main casting variables, such as PVDF concentration, SMM concentration and solvent evaporation time, on the surface properties of the synthesized membrane were identified and used to prepare optimal PVDF/SMM membrane. The prepared membranes were tested for permeation flux and salt rejection efficiency by membrane distillation process. The results indicate that the optimally modified membrane yields high permeate flux of around $17.5 \mathrm{~kg} / \mathrm{m}^{2} \mathrm{~h}$ and total salt rejection efficiency of $99.9 \%$ compared with pristine membranes. In particular, the hydrophobicity of the modified membrane significantly increases up to $108^{\circ}$ at water contact angle, through presented surface modification procedure.

Keywords: Surface-modifying macromolecules; Poly(vinylidene fluoride); Membrane modification; Membrane distillation; Response surface methodology

${ }^{*}$ Corresponding author. 